\title{
POLEMICAS
}




\section{EDUCACIÓN Y HEGEMONÍA EN EL NUEVO CAPITALISMO*}

JUAN CARLOS TEDESCO*

Director Instituto Internacional de Planeamiento de la Educación IIPE - UNESCO, Buenos Aires. j.tedesco@iipe-buenosaires.org.ar

\section{Resumen}

\section{Educación y hegemonía en el nuevo capitalismo: algunas notas e hipótesis de trabajo}

\section{JUAN CARLOS TEDESCO}

El texto analiza las nuevas modalidades de funcionamiento de las instituciones de socialización, particularmente la familia, los medios de comunicación y la escuela. Postula la hipótesis de la escasa vocación hegemónica del nuevo capitalismo y presenta los desafíos de la educación a partir de la idea según la cual la función de la escuela consiste en formar los marcos de referencia del sujeto para enfrentar los cambios permanentes a los cuales nos somete la producción cultural del nuevo capitalismo.

\section{Palabras claves}

Socialización, relaciones sociales, sociología de la educación

\section{Summary}

This text analyzes the new operation modalities of socialization institutions, particularly family, media and school. It postulates the hypothesis of the poor hegemonic vocation of new capitalism and it presents the challenges of education starting from the idea that the function of school is to form individual's reference marks needed to confront the permanent changes to which cultural production of new capitalism subjects us.

\section{Key words}

Socialization, social relationships, sociology of the education

\footnotetext{
* Este texto se pública con autorización de la Directora de la revista Propuesta Educativa de la FLACSO, Inés Dussel. Fue recibido el t de octubre de 2003 y arbitrado el 17 de octubre de 2003.

Director Instituto Internacional de Planeamiento de la Educación IIPE - UNESCO, Buenos Aires. J.tedesco@iipe-buenos aires.org.ar 


\section{Introducción}

Quisiera comenzar este artículo aclarando que la utilización del término "notas" en el título responde tanto a su forma como a su contenido. Estamos en un momento donde es necesario introducir una cuota significativa de prudencia en los análisis de problemas abiertos que, probablemente, admitan más de una respuesta. La necesidad de prudencia tiene que ver con la incertidumbre que provoca el desarrollo de los procesos sociales contemporáneos, acentuado a partir de los acontecimientos del 11 de septiembre. Pero si bien lo sucedido ese día obliga a revisar nuestros análisis de los procesos sociales, también nos obliga a adoptar mucha más seguridad y firmeza con respecto al carácter no negociable de ciertos valores y principios. Uno de los pilares de la educación del siglo XXI - "aprender a vivir juntos"- 1 ya no puede ser mantenido en el terreno de los discursos "blandos", de adhesión meramente retórica. Es preciso asumir que aprender (y, por lo tanto, enseñar) a vivir juntos, es tan importante como aprender a trabajar, a razonar, a experimentar o a emprender.

\section{La escasa vocación hegemónica del nuevo capitalismo}

En el capitalismo industrial, parafraseando a Durkheim, existía un alto nivel de "solidaridad orgánica". Más allá de la discusión acerca del organicismo en el análisis del orden social, lo cierto es que el capitalismo industrial era un capitalismo inclusivo que establecía vínculos entre todos los sectores sociales a través de relaciones de explotación y de dominación. El nuevo capitalismo ${ }^{2}$, en cambio, genera fenómenos de exclusión, que se definen, precisamente, por la mayor precariedad, la ausencia o la ruptura de los vínculos ${ }^{3}$. En este nuevo capitalismo, en consecuencia, vivir juntos no es una consecuencia "natural" del orden social. Vivir juntos sólo puede ser el producto de una decisión política, voluntaria, consciente, específicamente humana. Esta es una de las razones por las cuales, actualmente, las ciencias sociales tienden a asumir posiciones que ponen de manifiesto un fuerte resurgimiento del humanismo y de la filosofía social, frente a los paradigmas tradicionales del determinismo estructural.

El carácter inclusivo del capitalismo industrial implicaba la necesidad de postular un pensamiento hegemónico. La fuerte potencialidad excluyente del nuevo capitalismo, en cambio, debilita su vocación hegemónica. Esta afirmación puede resultar extraña cuando se habla tanto de "pensamiento único" y de ausencia de alternativas al modelo capitalista neoliberal. Sin embargo, es preciso no confundir pensamiento único con pensamiento hegemónico. El "pensamiento" neoliberal puede ser percibido como único porque hasta ahora no enfrenta alternativas, pero para ser hegemónico tiene que convencer y ser capaz de generar adhesión.

La debilidad de la vocación hegemónica del nuevo capitalismo está asociada a los nuevos patrones de funcionamiento de las instituciones responsables de la producción y la distribución de bienes culturales. En el capitalismo industrial, la cultura se basaba en instituciones que funcionaban sobre la base de la lógica de la oferta. La escuela $-y$ también la TV general- estaban basadas en ofrecer a todos un mismo producto y esta oferta tenía, por eso, un fuerte poder homogeneizador. En esta lógica y en este poder se expresaba la voluntad hegemónica de los sectores dominantes. Los nuevos mecanismos

\footnotetext{
${ }^{1}$ UNESCO. La educación encierra un tesoro. Informe para la UNESCO de la Comisión Internacional de Educación para el siglo XXI, presidida por Jacques Delors. UNESCO-Santillana, 1997.

${ }^{2}$ La expresión "nuevo capitalismo" está tomada de BOLTANSKY, Luc y CHIAPELLO, Eve. Le nouvel esprit du capitalisme. París, Gallimard, 1999.

${ }^{3}$ CASTEL, R. Metamorfosis de la cuestión social. Una crónica del salariado. Buenos Aires, Paidós, 1997.

Digitalizado por RED ACADEMICA
} 
culturales, en cambio, se basan mucho más en la lógica de la demanda. Internet, la TV por cable (y la escuela basada en los mecanismos de responder a las demandas del "alumno-cliente"), invierten el esquema existente en el capitalismo industrial y, en ese sentido, expresan la escasa vocación hegemónica del nuevo capitalismo.

En esta discusión, es oportuno retomar algunas de las hipótesis de Dominique Wolton ${ }^{4}$ acerca de las nuevas tecnologías de la información y, en particular, de Internet. Wolton nos advierte que poner el centro de la dinámica cultural en la demanda de los usuarios no es, necesariamente, un mecanismo más democrático que el basado en el control de la oferta. Para formular una demanda es necesario dominar los códigos de acceso al mundo. Al contrario de lo que sostiene el discurso actualmente dominante, la emancipación, el desarrollo personal, la libertad, pasan primero por la oferta, pues es ella la que permite constituir los marcos de referencia a partir de los cuales se podrá expresar, ulteriormente, la demanda. Wolton nos recuerda que uno de los efectos más importantes de la dominación sociocultural consiste, precisamente, en no pedir más que lo que uno ya tiene. La simple adecuación a la demanda, en última instancia, implica reforzar la dominación.

Una de las preguntas más importantes acerca de los actuales procesos de transformación social se refiere al carácter transitorio o permanente de algunos de sus rasgos más importantes. Esta pregunta tiene particular importancia cuando la referimos a la vocación hegemónica del sistema social capitalista globalizado. ¿Se trata de un fenómeno transitorio o, al contrario, en algún momento este nuevo capitalismo adoptará una configuración más estable y pretenderá perpetuarse a través de procesos clásicos de transmisión cultural? El libro reciente de M. Hardt y A. Negri ${ }^{5}$ plantea la hipótesis según la cual estaríamos ante un proceso de construcción de un nuevo orden social de carácter "imperial". De acuerdo a estos autores, ya se estarían conformando los elementos propios de una estructura consolidada, capaz de ejercer una función hegemónica y de recrear procesos de transmisión cultural regulada. Sin embargo, aun en estos análisis aparece la idea de que este "imperio" es decadente y que nace y se presenta a sí mismo como crisis y como un imperio de la corrupción. Los sucesos del 11 de septiembre han agudizado esta tensión y han puesto de relieve la relevancia de las opciones que estamos enfrentando: vivir juntos o entrar en la lógica fundamentalista donde el diferente es el enemigo.

A partir de estas consideraciones iniciales, es posible avanzar en el análisis de las relaciones entre escuela y cultura en el nuevo capitalismo. Esta relación se expresa no sólo a través de los contenidos que se transmiten sino de la lógica con la cual trabajan las instituciones que producen esos contenidos. Para avanzar en este análisis, haremos referencia a los dos ámbitos institucionales más importantes donde tiene lugar el proceso de transmisión cultural: la familia y la escuela.

\section{Cambios en la familia}

Como se sabe, la descripción clásica del proceso de socialización distingue la existencia de dos fases principales: la socialización primaria y la socialización secundaria ${ }^{6}$. La primera, efectuada durante la niñez y habitualmente en el seno de la familia, es aquella por la que el individuo adquiere el lenguaje, los esquemas básicos de interpretación de la realidad y los rudimentos del aparato legitimador de dicha realidad. La segunda, en

\footnotetext{
${ }^{4}$ WOLTON, Dominique. Internet y después? Buenos Aires, Gedisa, 2000.

${ }^{5}$ Ver HARDT, M. y NEGRI, A. Empire. París, Exils Ed. 2000.

${ }^{6}$ BERGER, P. y LUCKMAN, Th. La construcción social de la realidad. Buenos Aires, Amorrortu, 1995. 
cambio, es todo proceso posterior que introduce al individuo ya socializado a nuevos sectores del mundo objetivo de su sociedad. Los análisis del proceso de socialización también destacaron que las dos características más importantes de la socialización primaria eran la carga afectiva con la cual se transmiten sus contenidos y la identificación absoluta con el mundo tal como lo presentan los adultos.

Estas características de la familia y del proceso de socialización, sin embargo, están sufriendo modificaciones significativas. Por un lado, se registran cambios muy importantes en la composición familiar. Al respecto, los datos disponibles para la mayoría de los países de cultura occidental indican una serie de fenómenos muy importantes: expansión de la familia nuclear, reducción del número de hijos, crecimiento de las uniones libres y del número de hijos que viven sólo con uno de sus progenitores (mayoritariamente la madre), ausencia de la figura paterna o cambio frecuente de dicha figura, disociación entre padre biológico y padre psicológico y modificaciones muy importantes en los roles del padre y de la madre en el seno del comportamiento familiar. En síntesis, el cambio fundamental que se ha producido en la familia es la disociación entre conyugalidad (vínculo de pareja) y filiación (vínculos entre padres/madres e hijos/hijas). En la familia tradicional, estos dos vínculos eran indisolubles. En la actualidad, en cambio, mientras la conyugalidad ha perdido su carácter indisoluble, porque es una relación social, la filiación mantiene dicho carácter porque es un vínculo natural. El proceso de secularización implicó, desde este punto de vista, un proceso de transformación de las relaciones de pareja en relaciones temporales y más igualitarias.

Pero además de estos cambios en la estructura y composición de la familia, también se aprecian cambios importantes en los contenidos y en las formas como dichos contenidos son transmitidos. Diversos estudios indican que son cada vez más los adultos que consideran que no deben transmitir a sus hijos una determinada visión del mundo sino la capacidad para elegir y formar autónomamente su propia concepción. La familia contemporánea tiene tendencia a asumir la forma de una "red de relaciones" que, en lugar de ser responsable de transmitir el patrimonio cultural y moral de una generación a otra, tiende ahora a privilegiar la construcción de la identidad personal ${ }^{7}$.

Z. Laïdi, uno de los más interesantes analistas franceses contemporáneos del proceso de globalización, sostiene la hipótesis según la cual estaríamos ante una crisis de la función de transmisión en la sociedad occidental contemporánea. Evocando los análisis de Hanna Arendt y de Pierre Legendre, Laïdi constata que la transmisión resulta de una articulación entre tres elementos: (i) el reconocimiento de una referencia absoluta que crea una obligación con respecto a las generaciones futuras; (ii) la existencia de ámbitos institucionales donde dicha obligación tiene lugar, y (iii) la aceptación de ciertas mediaciones que sólo tienen sentido porque la transmisión se inscribe en una dimensión de largo plazo. La crisis de la transmisión en la sociedad actual se produce, según Laïdi, porque ninguna de estas condiciones logra mantener su vigencia.

La transmisión efectuada en el seno de la familia ya no se apoya en la aceptación del principio de autoridad, en la existencia de una paternidad simbólica, sino que el único principio válido es el principio democrático o, dicho en otros términos, la relativización de las opiniones. La paradoja de esta situación, según Laïdi, es que la democracia se extiende a la vida privada y al ámbito de los estilos de vida, al mismo tiempo que pierde

\footnotetext{
7 THÉRY, Irène. "Différence des sexes et différence des générations; l'institution familiale en déshérence", en Esprit, $\mathrm{n}^{\circ}$ 227, décembre de 1996. Para el caso de España, ver Gerardo Meil Landwerlin. La postmodernización de la familia española. Madrid, Acento Editorial, 1999. También el estudio de PÉREZ ALONSO-GETA, Petra María. El Magisterio Español, 3 de enero de 1996 
importancia en el ámbito de la vida pública. Para decirlo en palabras del propio Laïdi, " la lógica ciudadana coloniza el espacio privado en el mismo momento en que parece desertar del espacio público". Si la familia se transforma en una red integrada por individuos solidarios pero iguales, ya no hay transmisión sino intercambio. Las preguntas que esta situación provoca son decisivas para las relaciones entre generaciones, tanto para las que tienen lugar entre padres e hijos, como entre adultos y jóvenes y maestros y alumnos: ¿como pensar la transmisión en una sociedad que no reconoce otro valor más que el valor de cambio? ¿ Que sentido otorgar a la idea de transmisión en una sociedad donde el saber es pensado y percibido como algo cada vez más fluido, amenazado de obsolescencia permanente y medido en función de su utilidad económica? ${ }^{8}$.

La crisis de la función de transmisión inter-generacional está asociada a una de las características más importante del contexto cultural del nuevo capitalismo: la concentración en el presente. Las transformaciones son tan profundas, que se viven como una ruptura con el pasado. Se diluye la idea de continuidad histórica y todo aparece como nuevo y fundacional. Al respecto, ya en 1970, Margaret Mead efectuaba un análisis que vale la pena recordar hoy, tanto por su enfoque como por el carácter testimonial de la percepción que desde las ciencias sociales se tenía del fenómeno de la transmisión cultural. M. Mead distinguía tres modelos de transmisión, el prefigurativo, el cofigurativo y el postfigurativo. El primero, en el cual los adultos conocen y enseñan los valores de la sociedad a las nuevas generaciones, corresponde a las sociedades llamadas tradicionales. El segundo, donde adultos y jóvenes aprenden al mismo tiempo porque todo es nuevo para ambos, corresponde a sociedades que pasan por una etapa revolucionaria o viven procesos masivos de migración e incorporación a nuevas culturas. El tercero, que según $\mathrm{M}$. Mead corresponde a la sociedad actual, es aquel donde los jóvenes son quienes saben y quienes enseñan. Aunque sea un poco extensa, me parece pertinente citar sus propias palabras:

"Nuestra crisis actual ha sido atribuida tanto a la abrumadora celeridad del cambio, como al derrumbe de la familia, a la decadencia del capitalismo, al triunfo de la tecnología sin alma y, en términos de repudio total, a la quiebra definitiva del Sistema (Establishment). Detrás de estos asertos se observa un conflicto más fundamental entre aquellos para quienes el presente sólo encarna una intensificación de nuestra cultura cofigurativa ya existente, en la que los pares están reemplazando cada vez más a los padres como modelos significativos de conducta, y aquellos que alegan que en verdad estamos ingresando en una etapa totalmente nueva de la evolución cultural." (...). "Actualmente en ningún lugar del mundo hay mayores que sepan lo que saben los jóvenes, por muy remotas y sencillas que sean las sociedades donde viven estos últimos. Antaño siempre había algunos adultos que sabían más que cualquier joven en términos de la experiencia adquirida al desarrollarse dentro de un sistema cultural. Ahora no los hay. No se trata sólo de que los padres ya no son guías sino de que no existen guías, los busque uno en su propio país o en el extranjero. No hay adultos que sepan lo que saben acerca del mundo en que nacieron quienes se han criado dentro de los últimos veinte años." (...) "...los adultos de hoy deben interpretar que su propio pasado es incomunicable y deben enseñar a sus hijos, por mucho que ello les duela, que no tienen que interrogarlos, porque nunca podrán entender. Necesitamos convencernos de que ninguna otra generación experimentará jamás lo que hemos experimentado nosotros. Desde este punto de vista hemos de reconocer que no tenemos descendientes, del mismo modo que nuestros hijos no tienen antepasados. En este punto de ruptura entre dos grupos radicalmente distintos e íntimamente vinculados, es inevitable que ambos estén muy solos, mientras nos

\footnotetext{
${ }^{8}$ LAIIDI, Zaidi. Le sacre du présent. Paris, Flammarion, 2000.
} 
miramos los unos a los otros seguros de que ellos nunca experimentarán lo que hemos experimentado nosotros y que nosotros nunca podremos experimentar lo que han experimentado ellos." 9

La visión de Margaret Mead estaba obviamente influida por las características de los movimientos juveniles de la década 1960-1970. La ruptura con el pasado no abrió las perspectivas a un futuro distinto sino a una significativa ausencia de futuro. La incertidumbre, la ausencia de óptimos fijos, la idea que nada es ni será estable, provoca serias dificultades para elaborar cualquier perspectiva de larga duración. Las consecuencias que provoca esta concentración en el presente en la formación de la personalidad y en las relaciones sociales han comenzado a ser percibidas y analizadas en los últimos años. Richard Sennett ${ }^{10}$ nos ha ofrecido un estudio cualitativo muy importante referido a la situación de sectores incluidos en el nuevo capitalismo. La concentración en el presente o, en términos de Sennett, el "nada a largo plazo", es un principio que "corroe la confianza, la lealtad y el compromiso mutuos". En este sentido, la dinámica temporal del mundo del trabajo y de la vida ciudadana, donde todo se juega en el presente, genera un conflicto básico con la dinámica de la vida familiar, donde tiene un lugar importante lo intemporal, lo permanente y lo absoluto. El problema, sostiene Sennett, pasa a ser ahora "...cómo proteger las relaciones familiares para que no sucumban a los comportamientos a corto plazo, el modo de pensar inmediato y, básicamente, el débil grado de lealtad y compromiso que caracterizan al moderno lugar de trabajo"11.

Los cambios en el proceso de transmisión cultural -des-institucionalización de la familiaabren la puerta a un conjunto de fenómenos sociales complejos y diferentes, según los contextos sociales y culturales. Alain Touraine se ocupó de señalar las posibilidades que abría este proceso para la construcción del Sujeto ${ }^{12}$. Pero el mismo Touraine advertía sobre las "protecciones institucionales" que requerían estos procesos de construcción. El problema es que asistimos precisamente a una erosión significativa de las bases institucionales con las cuales funciona la sociedad, particularmente visible en las sociedades 0 en los sectores sociales afectados por procesos de precarización y de exclusión. Los nuevos sistemas institucionales parecen basarse en la idea de no hacerse cargo del destino de las personas. Cuando las condiciones materiales no aseguran esta capacidad de ejercer autónomamente la posibilidad de definir un proyecto y elegir las opciones más apropiadas, lo que presenciamos es la generalización de la anomia, la ingobernabilidad y la apelación a las formas mas tradicionales de dominación. En estos contextos no es casual que se vuelva a postular el problema de la educabilidad como concepto necesario para analizar situaciones que se presentan en países o en sectores sociales afectados por niveles significativos de exclusión social ${ }^{13}$.

\section{Nuevo papel de la escuela}

Todo el análisis anterior apunta a la idea según la cual la formación de los marcos de referencia con los cuales cada persona procesa los mensajes que recibe se convierten, por ello, en el elemento central del desempeño ciudadano. El punto central en el cual quisiera concentrar las reflexiones que siguen es que la función de la escuela en relación

\footnotetext{
${ }^{9}$ MEAD, Margaret. Cultura y compromiso. El mensaje de la nueva generación. Barcelona, Gedisa, 1997.

10 SENNETT, R. La corrosión del carácter. Las consecuencias personales del trabajo en el nuevo capitalismo. Barcelona, Anagrama, 2000.

11 Ibíd., pág. 25.

12 TOURAINE, Alain. Crítica de la modernidad. Buenos Aires, Fondo de Cultura Económica, 1994. Id. ¿Podremos vivir juntos? Iguales y diferentes. Buenos Aires, Fondo de Cultura Económica, 1997.

13 TEDESCO, Juan Carlos. Educar en la sociedad del conocimiento. Buenos Aires, Fondo de Cultura Económica, 2000.
} 
a la cultura consiste en la formación del núcleo estable, de los marcos de referencia, que permitirán enfrentar los cambios permanentes a los cuales nos somete la producción cultural del nuevo capitalismo. Dichos marcos de referencia son tanto culturales como cognitivos. Desde el punto de vista cultural, las informaciones y las opciones de conductas son procesadas a través de una serie de operaciones de identificación, de reconocimiento, de diferenciación, de adhesión o de rechazo, que suponen la existencia de un núcleo cultural básico, desde el cual es posible elegir y responder a los mensajes culturales. Desde el punto de vista cognitivo sucede algo similar: el acceso a las informaciones provoca procesos de comparación, asociación, transferencia, etc. que dependen del desarrollo intelectual del sujeto. Cuando este núcleo cultural y cognitivo no está constituido o lo está muy débilmente, los riesgos de alienación y de dependencia aumentan considerablemente, ya que los medios de comunicación, particularmente la televisión, no han sido concebidos para formar este núcleo. La oferta de los nuevos medios y agencias de socialización supone que los usuarios ya tienen las categorías y las capacidades de observación, de clasificación, de comparación, etc., necesarias para procesar e interpretar el enorme caudal de datos que ellos ponen a nuestra disposición.

Cuáles debe ser los contenidos de este núcleo "duro" del desarrollo cognitivo y cultural y cómo se deciden, constituyen un motivo de debate muy importante. En realidad, el eje que divide las posiciones en este campo pasa por decidir si los contenidos de ese núcleo duro deben ser discutidos socialmente o deben ser decididos en forma individual y privada. En la medida que los aparatos culturales del pasado actuaban desde la oferta y en esa oferta el Estado tenía un papel preponderante, la discusión sobre el contenido de los marcos de referencia asumía cierto carácter público. Las nuevas modalidades de producción cultural están, en cambio, basadas en tecnologías manejadas por grandes consorcios de empresas privadas que actúan siguiendo la lógica del beneficio a corto plazo y del control de las demandas de la población consumidora. El interrogante y el desafío que se abre en este nuevo contexto pasa por definir modalidades de participación alternativas al autoritarismo del control estatal y al individualismo a-social de la lógica privada.

Desde el punto de vista de los contenidos de los marcos de referencia, sería posible sintetizarlos en dos de los pilares de la educación del siglo XXI, definidos en el informe de la comisión de la UNESCO, presidida por Jacques Delors: aprender a aprender y aprender a vivir juntos ${ }^{14}$. No parece necesario describir aquí el contenido de estos ejes de acción, que responden a la dimensión cognitiva el primero y a la cultural el segundo. Lo que interesa destacar es, en última instancia, que el desarrollo de esos pilares supone introducir en la escuela la posibilidad de vivir experiencias que no se producen "naturalmente" en el espacio externo a la escuela. Aprender a aprender implica un esfuerzo de reflexión sobre las propias experiencias de aprendizaje que no pueden desarrollarse sin un guía, sin un modelo, sin un "acompañante cognitivo" 15 , que sólo la actividad educativa organizada puede proporcionar. Aprender a vivir juntos, por su parte, implica vivir experiencias de contacto con el diferente, experiencias de solidaridad, de respeto, de responsabilidad con respecto al otro, que la sociedad no proporciona naturalmente. La escuela puede, en este sentido, recuperar su función cultural a través del desarrollo de experiencias que no tienen lugar en la cultura externa. Dicho de otra manera, la escuela puede cumplir un papel cultural y social significativo si asume un cierto grado de tensión y conflicto con la cultura. Su papel no es "adecuarse" a la cultura

\footnotetext{
${ }^{14}$ UNESCO. La educación encierra un tesoro. Op. Cit.

${ }^{15}$ Ver: Goery Delacôte Enseñar y aprender con nuevos métodos. La revolución cultural de la era electrónica. Barcelona, Gedisa, 1997.
} 
popular, ni tampoco, por supuesto, aislarse ni vaciarse de contenidos por la vía del empobrecimiento de los contenidos que ella transmite.

Las condiciones para que la escuela pueda cumplir este papel son tanto institucionales como pedagógicas. El principio básico radica en la idea de autonomía para el desarrollo de propuestas curriculares. Pero esta cuestión de la autonomía debe ser objeto de un cuidadoso análisis contextual. La historia reciente de las transformaciones educativas muestra que la idea de la autonomía puede ser defendida desde posiciones opuestas, que oscilan entre la autonomía como des-responsabilización por parte del Estado (posición visible en las reformas educativas de muchos países pobres, donde este concepto fue el argumento para bajar los costos y el papel del Estado en el financiamiento de la educación, con consecuencias claramente regresivas desde el punto de vista de la distribución de la oferta educacional) hasta la autonomía como parte de un proyecto político-educativo destinado a permitir mayor participación de las familias y de los docentes en la elaboración de proyectos pedagógicos pertinentes a las necesidades básicas de aprendizaje de los alumnos. En todo caso, la idea central consiste en recuperar el carácter de la escuela como espacio donde es posible programar experiencias discutidas socialmente. Esta alternativa se opone tanto a la salida individual a-social, de los que promueven la idea de educarse en el seno de la familia y a través de mecanismos basados en las nuevas tecnologías de la información como la de aquellos que promueven una escuela supeditada a los dictámenes del poder estatal, uniforme y promotora de un sólo modo de ver y de concebir el mundo.

De este análisis se desprende una conclusión obvia, referida a la centralidad de los docentes como actores sociales y como profesionales de los procesos de transmisión cultural. La literatura sobre este tema es abundante y apunta a comprender las raíces de lo que se ha dado en llamar el "malestar docente". Dicho malestar, que atraviesa situaciones objetivas muy diferentes, tiene más que ver con la crisis de transmisión a la que nos referimos en los puntos anteriores que con situaciones vinculadas a condiciones materiales de trabajo.

Obviamente, estas reflexiones sobre el papel de la educación y de la escuela pueden ser consideradas como ingenuamente voluntaristas. ¿Cómo podría la educación y sus formas institucionales tradicionales desarrollar una acción contracultural tan importante como la aquí se sugiere? Responder a esta pregunta escapa a los límites de estas notas, que sólo llegan a plantearla. Sin embargo, es importante al menos admitir la legitimidad de un postulado voluntarista. Dicha legitimidad proviene tanto de un enfoque filosófico como sociopolítico. Desde el punto de vista filosófico, ya ha sido desarrollada la idea según la cual la característica básica de la condición humana es la posibilidad de superar los determinismos de orden natural (herencia genética) o de orden social y cultural (origen social, étnico, religioso, etc.). Al respecto, me parece interesante retomar los aportes de Luc Ferry $^{16}$, quien asume que nuestros comportamientos -particularmente nuestros comportamientos morales- no pueden ser explicados por factores genéticos ni por determinantes socioculturales. Lo humano implica trascender dichos determinantes. Si la naturaleza o la historia fueran nuestro código, no sería posible cuestionar el mundo, juzgarlo, transformarlo, inventarlo. Si yo argumentara siempre como argentino, francés o español, como originario de tal familia o de tal sexo, o como perteneciente a tal grupo, sector o clase social estaría, por definición, encerrado en los comunitarismos naturales o étnicos y sería incapaz de proyectarme más allá de mi condición para acceder a cualquier forma de universalidad. Pero el voluntarismo tiene, además, una justificación sociopolítica

${ }^{16}$ COMPTE-SPONVILLE, André y FERRY, Luc. La sagesse des modernes: Dix questions pour notre temps. Paris, Laffont, 1998.

Digitalizado por RED ACADEMICA 
que proviene del alto nivel de reflexividad del comportamiento ciudadano en la sociedad moderna. Sabemos que la mayor reflexividad no implica mayor control de las situaciones sino mayores niveles de incertidumbre y riesgo ${ }^{17}$. Pero ese espacio abierto es un espacio de indeterminación que abre mayores niveles de autonomía a los sujetos y, en ese mismo sentido, mayores niveles de responsabilidad. En otras palabras, el determinismo no puede ser un argumento para evitar asumir la responsabilidad por nuestro destino.

17 GIDDENS, A. Consecuencias de la modernidad. Madrid, Alianza, 1997. 